\title{
On Global Asymptotic Stability of Neural Networks with Discrete and Distributed Delays
}

\author{
Zidong Wang, Yurong Liu and Xiaohui Liu
}

\begin{abstract}
In this paper, the global asymptotic stability analysis problem is investigated for a class of neural networks with discrete and distributed time-delays. The purpose of the problem is to determine the asymptotic stability by employing some easy-to-test conditions. It is shown, via the Lyapunov-Krasovskii stability theory, that the class of neural networks under consideration are globally asymptotically stable if a quadratic matrix inequality involving several parameters is feasible. Furthermore, a linear matrix inequality (LMI) approach is exploited to transform the addressed stability analysis problem into a convex optimization problem, and sufficient conditions for the neural networks to be globally asymptotically stable are then derived in terms of a linear matrix inequality, which can be readily solved by using the Matlab LMI toolbox. Two numerical examples are provided to show the usefulness of the proposed global stability condition.
\end{abstract}

\section{Keywords}

Neural networks; Distributed delays; Discrete delays; Lyapunov-Krasovskii functional; Global asymptotic stability; Linear matrix inequality.

\section{INTRODUCTION}

Recently, it has been well recognized that time delays are often encountered in various neural networks, such as Hopfield neural networks, cellular neural networks and bi-directional associative memory networks, and the time delays are often the sources of oscillation and instability of neural networks [1], [2], [5], [7], [25], [26]. Therefore, the dynamical characteristics (including stable, unstable, oscillatory, and chaotic behavior) of neural networks with time delays have become a subject of intense research activities. In particular, since global stability is one of the most desirable dynamic properties of neural networks, there have been growing research interests on the stability analysis problem for delayed neural networks, and a large amount of literature has been available. Sufficient conditions, either delay-dependent or delay-independent, have been proposed to guarantee the asymptotic or exponential stability for neural networks, see [1], [5], [12], [13], [7], [19], [20], [22] for some recent results. It is noticed that, so far, most works on delayed neural networks have dealt with the stability analysis problem for neural networks with discrete time-delays.

On the other hand, as pointed out in [8], [16], [18], neural networks usually have a spatial extent due to the presence of a multitude of parallel pathways with a variety of axon sizes and lengths, and hence there is a distribution of propagation delays over a period of time. For example, in [18], a neural circuit has been designed with distributed delays, which solves a general problem of recognizing patterns in a time-dependent

This work was supported in part by the Engineering and Physical Sciences Research Council (EPSRC) of the U.K. under Grant GR/S27658/01, the Nuffield Foundation of the U.K. under Grant NAL/00630/G, and the Alexander von Humboldt Foundation of Germany.

Z. Wang was with the School of Information Sciences and Technology, Donghua University, Shanghai 200051, China, and is now with the Department of Information Systems and Computing, Brunel University, Uxbridge, Middlesex, UB8 3PH, U.K. Email: Zidong. Wang@brunel.ac.uk, Fax: $++44 / 1895251686$.

Y. Liu is with the Department of Mathematics, Yangzhou University, Yangzhou 225002, P. R. China.

$\mathrm{X}$. Liu is with the Department of Information Systems and Computing, Brunel University, Uxbridge, Middlesex, UB8 3PH, U.K. 
signal. It is noticed that, although the signal propagation is sometimes instantaneous and can be modeled with discrete delays, it may also be distributed during a certain time period so that the distributed delays should be incorporated in the model. In other words, it is often the case that the neural network model possesses both discrete and distributed delays [17].

Very recently, there have been some initial studies on the stability analysis issue for various neural networks with distributed time-delays, see [14], [23], [24], [27], [28]. In [27], by employing several Lyapunov functionals, the global exponential stability and asymptotic stability of generalized neural networks with distributed delays have been investigated, and a number of sufficient conditions for these types of stability have been derived. The global asymptotic stability has been studied in [14] for bi-directional associative memory (BAM) networks with distributed delays. In [23], criteria ensuring the existence, uniqueness, and global asymptotic stability have been derived for Hopfield neural networks involving distributed delays. In [24], the existence and global attractivity of the almost periodic solution have been examined for a class of cellular neural network with distributed delays and variable coefficients.

It should be pointed out that, despite the importance of both discrete and distributed delays in modeling neural networks, the stability analysis problem for general neural networks with discrete and distributed delays has not received much research attention. It is noted that, in [17], a two-neuron network model with multiple discrete and distributed delays has been studied, and local stability of the steady-state solutions and the oscillation around the steady-state solutions have been investigated. Unfortunately, the results in [17] cannot be directly applied for general neural networks. Up to now, there have been very few results on the analysis issue for the global asymptotic stability of general neural networks with discrete and distributed delays, which remains important and challenging.

In this paper, we aim to tackle the global asymptotic stability analysis problem for a class of neural networks with discrete and distributed time-delays. By employing a Lyapunov-Krasovskii functional and some new techniques, the addressed stability analysis problem is converted into a convex optimization problem. Then, different from commonly used matrix norm theories (such as $M$-matrix method), we utilize a linear matrix inequality (LMI) approach to establish sufficient conditions for the neural networks to be globally asymptotically stable. Note that LMIs can be easily solved by using the Matlab LMI toolbox, and no tuning of parameters is required [3]. Two numerical examples are provided to show the usefulness of the proposed global stability condition.

Notations: The notations are quite standard. Throughout this paper, $\mathbb{R}^{n}$ and $\mathbb{R}^{n \times m}$ denote, respectively, the $n$-dimensional Euclidean space and the set of all $n \times m$ real matrices. The superscript " $T$ " denotes matrix transposition and the notation $X \geq Y$ (respectively, $X>Y$ ) where $X$ and $Y$ are symmetric matrices, means that $X-Y$ is positive semidefinite (respectively, positive definite). $I_{n}$ is the $n \times n$ identity matrix. $|\cdot|$ is the Euclidean norm in $\mathbb{R}^{n}$. If $A$ is a matrix, denote by $\|A\|$ its operator norm, i.e., $\|A\|=\sup \{|A x|:|x|=1\}=$ $\sqrt{\lambda_{\max }\left(A^{T} A\right)}$ where $\lambda_{\max }(\cdot)$ (respectively, $\lambda_{\min }(\cdot)$ ) means the largest (respectively, smallest) eigenvalue of $A$. $l_{2}[0, \infty]$ is the space of square integrable vector. Denote by $L_{\mathcal{F}_{0}}^{p}\left([-h, 0] ; \mathbb{R}^{n}\right)$ the family of all $\mathcal{F}_{0}$-measurable $C\left([-h, 0] ; \mathbb{R}^{n}\right)$-valued variables $\xi=\{\xi(\theta):-h \leq \theta \leq 0\}$ such that $\sup _{-h \leq \theta \leq 0}|\xi(\theta)|^{p}<\infty$. The shorthand $\operatorname{diag}\left\{M_{1}, M_{2}, \cdots, M_{N}\right\}$ denotes a block diagonal matrix with diagonal blocks being the matrices $M_{1}, M_{2}, \cdots$, $M_{N}$. Sometimes, the arguments of a function or a matrix will be omitted in the analysis when no confusion can arise. 


\section{PROBLEM FORMULATION}

Consider the following delayed neural network with $n$ neurons:

$$
\dot{u}(t)=-A u(t)+W_{0} g_{0}(u(t))+W_{1} g_{1}(u(t-h))+W_{2} \int_{t-\tau}^{t} g_{2}(u(s)) d s+V
$$

where $u(t)=\left[u_{1}(t), u_{2}(t), \cdots, u_{n}(t)\right]^{T} \in \mathbb{R}^{n}$ is the state vector of the neural network, $A=\operatorname{diag}\left(a_{1}, a_{2}, \cdots, a_{n}\right)$ is a diagonal matrix with positive entries $a_{i}>0$. $W_{0}=\left(w_{i j}^{0}\right)_{n \times n}, W_{1}=\left(w_{i j}^{1}\right)_{n \times n}$, and $W_{2}=\left(w_{i j}^{2}\right)_{n \times n}$ are, respectively, the connection weight matrix, the discretely delayed connection weight matrix, and the distributively delayed connection weight matrix. $g_{i}(u(t))=\left[g_{i 1}\left(u_{1}\right), g_{i 2}\left(u_{2}\right), \cdots, g_{i n}\left(u_{n}\right)\right]^{T}$ denotes the neuron activation function with $g_{i}(0)=0$, and $V=\left[V_{1}, V_{2}, \cdots, V_{n}\right]^{T}$ is a constant vector. The scalar $h>0$, which may be unknown, denotes the discrete time delay, whereas the scalar $\tau>0$ is the known distributed time-delay.

It is usually assumed that the activation functions are continuous, differentiable, monotonically increasing and bounded, such as the sigmoid-type of function. As discussed in [7], [15], in many electronic circuits, the input-output functions of amplifiers may be neither monotonically increasing nor continuously differentiable, hence nonmonotonic functions can be more appropriate to describe the neuron activation in designing and implementing an artificial neural network. In this paper, we assume we make following assumptions for the neuron activation functions.

Assumption 1: The neuron activation functions in $(1), g_{i}(\cdot)$, satisfy the following Lipschitz condition

$$
\left|g_{i}(x)-g_{i}(y)\right| \leq\left|G_{i}(x-y)\right|, \quad(i=0,1,2)
$$

where $G_{i} \in \mathbb{R}^{n \times n}$ are known constant matrices.

Assumption 2: The neuron activation functions in (1) are bounded.

Remark 1: The type of activation functions in (2) is not necessarily monotonic and smooth, and have been used in numerous papers, see [7] and references therein.

Remark 2: Usually, various fixed point theorems such as Brouwer's fixed point theorem, Schauder fixed point theorem and contraction mapping principle can be exploited to prove the existence of equilibrium points of neural networks. For example, under Assumption 2, it is not difficult to ensure the existence of equilibrium point of the system (1) by using Brouwer's fixed point theorem. In the sequel we shall analyze the global asymptotic stability of the equilibrium point, which in turn implies the uniqueness of the equilibrium point.

To simplify the asymptotic stability analysis of (1), we let $u^{*}$ be the equilibrium point of (1), and shift the intended equilibrium $u^{*}$ to the origin by letting $x=u-u^{*}$, and then the system (1) can be transformed into:

$$
\dot{x}(t)=-A x(t)+W_{0} l_{0}(x(t))+W_{1} l_{1}(x(t-h))+W_{2} \int_{t-\tau}^{t} l_{2}(x(s)) d s,
$$

where $x(t)=\left[x_{1}(t), x_{2}(t), \cdots, x_{n}(t)\right]^{T} \in \mathbb{R}^{n}$ is the state vector of the transformed system. It follows from (2) that the transformed neuron activation functions

$$
l_{i}(x)=g_{i}\left(x+u^{*}\right)-g_{i}\left(u^{*}\right) \quad(i=0,1,2)
$$

satisfy

$$
\left|l_{i}(x)\right| \leq\left|G_{i} x\right|
$$

where $G_{i} \in \mathbb{R}^{n \times n}(i=0,1,2)$ are specified in (2). Notice that we do not need the traditional monotonicity and smoothness assumptions on the activation function $l_{i}(x)$. 
Before stating our main purpose in this paper, let us introduce the notion of the global asymptotic stability for the neural network (3). Now, let $x(t ; \xi)$ denote the state trajectory of the neural network (3) from the initial data $x(\theta)=\xi(\theta)$ on $-h \leq \theta \leq 0$ in $L_{\mathcal{F}_{0}}^{2}\left([-h, 0] ; \mathbb{R}^{n}\right)$. It can be easily seen that the system (3) admits a trivial solution $x(t ; 0) \equiv 0$ corresponding to the initial data $\xi=0$.

Definition 1: [4], [10] The equilibrium point (trivial solution) 0 is said to be globally asymptotically stable if it is locally stable in the sense of Lyapunov and global attractive, where global attractivity means that every trajectory tends to the equilibrium point as $t \rightarrow \infty$.

The main aim of this paper is to establish LMI-based sufficient conditions under which the global asymptotic stability is guaranteed for the neural network (3) with both discrete and distributed time delays.

\section{MAIN RESULTS AND PROOFS}

The following lemmas are essential in establishing our results by using the LMI approach.

Lemma 1: [3] Given constant matrices $\Omega_{1}, \Omega_{2}, \Omega_{3}$ where $\Omega_{1}=\Omega_{1}^{T}$ and $0<\Omega_{2}=\Omega_{2}^{T}$, then

$$
\Omega_{1}+\Omega_{3}^{T} \Omega_{2}^{-1} \Omega_{3}<0
$$

if and only if

$$
\left[\begin{array}{cc}
\Omega_{1} & \Omega_{3}^{T} \\
\Omega_{3} & -\Omega_{2}
\end{array}\right]<0, \quad \text { or } \quad\left[\begin{array}{cc}
-\Omega_{2} & \Omega_{3} \\
\Omega_{3}^{T} & \Omega_{1}
\end{array}\right]<0 .
$$

Lemma 2: [9] For any positive definite matrix $M>0$, scalar $\gamma>0$, vector function $\omega:[0, \gamma] \rightarrow \mathbb{R}^{n}$ such that the integrations concerned are well defined, the following inequality holds:

$$
\left(\int_{0}^{\gamma} \omega(s) d s\right)^{T} M\left(\int_{0}^{\gamma} \omega(s) d s\right) \leq \gamma\left(\int_{0}^{\gamma} \omega^{T}(s) M \omega(s) d s\right)
$$

We are now ready to derive the conditions under which the network dynamics of (3) is globally asymptotically stable. The following theorem reveals that such conditions can be expressed in terms of the positive definite solution to a quadratic matrix inequality involving several scalar parameters.

Theorem 1: If there exist positive scalars $\varepsilon_{1}>0, \varepsilon_{2}>0, \varepsilon_{3}>0$ and a positive definite matrix $P>0$ such that the following quadratic matrix inequality

$$
\begin{gathered}
-A P-P A+P\left(\varepsilon_{1}^{-1} W_{0} W_{0}^{T}+\varepsilon_{2}^{-1} W_{1} W_{1}^{T}+\varepsilon_{3}^{-1} W_{2} W_{2}^{T}\right) P \\
+\varepsilon_{1} G_{0}^{T} G_{0}+\varepsilon_{2} G_{1}^{T} G_{1}+\varepsilon_{3} \tau^{2} G_{2}^{T} G_{2}<0
\end{gathered}
$$

holds, then the dynamics of the neural network (3) is globally asymptotically stable.

Proof: It follows immediately from (4) that

$$
l_{i}^{T}(t) l_{i}(t) \leq\left|G_{i} x\right|^{2}=x^{T} G_{i}^{T} G_{i} x .
$$

In order to establish the stability conditions, we introduce the following Lyapunov functional candidate

$$
\Phi(x(t))=x^{T}(t) P x(t)+\int_{t-h}^{t} x^{T}(s) Q_{1} x(s) d s+\int_{-\tau}^{0} \int_{t+s}^{t} e^{T}(\eta) Q_{2} e^{T}(\eta) d \eta d s,
$$

where $P$ is the positive definite solution to the inequality (6), and $Q_{1} \geq 0$ and $Q_{2} \geq 0$ are defined, respectively, by

$$
\begin{aligned}
Q_{1} & :=\varepsilon_{2} G_{1}^{T} G_{1}, \\
Q_{2} & :=\varepsilon_{3} \tau G_{2}^{T} G_{2} .
\end{aligned}
$$


The time derivative of $\Phi(x(t))$ along a given trajectory is calculated as follows:

$$
\begin{aligned}
\frac{d \Phi(x(t))}{d t}= & x^{T}(t)\left(-A P-P A+Q_{1}+\tau Q_{2}\right) x(t)+x^{T}(t) P W_{0} l_{0}(t)+l_{0}^{T}(t) W_{0}^{T} P x(t) \\
& +x^{T}(t) P W_{1} l_{1}(t-h)+l_{1}^{T}(t-h) W_{1}^{T} P x(t) \\
& -x^{T}(t-h) Q_{1} x(t-h)+e^{T}(t) P W_{2} \int_{t-\tau}^{t} l_{2}(s) d s \\
& +\left(\int_{t-\tau}^{t} l_{2}(s) d s\right)^{T} W_{2} P x(t)-\int_{t-\tau}^{t} e^{T}(s) Q_{2} x(s) d s .
\end{aligned}
$$

Let $\varepsilon_{1}>0, \varepsilon_{2}>0, \varepsilon_{3}>0$ be three positive scalars, and denote

$$
\Delta:=\left[\begin{array}{c}
\varepsilon_{1}^{1 / 2} l_{0}(t)-\varepsilon_{1}^{-1 / 2} W_{0}^{T} \operatorname{Px}(t) \\
\varepsilon_{2}^{1 / 2} l_{1}(t-h)-\varepsilon_{2}^{-1 / 2} W_{1}^{T} \operatorname{Px}(t) \\
\left.\varepsilon_{3}^{1 / 2} \int_{t-\tau}^{t} l_{2}(s) d s-\varepsilon_{3}^{-1 / 2} W_{2}^{T} \operatorname{Px}(t)\right)
\end{array}\right] .
$$

Then, it follows from the matrix inequality $\Delta^{T} \Delta \geq 0$ and the relation (7) that

$$
\begin{aligned}
& x^{T}(t) P W_{0} l_{0}(t)+l_{0}^{T}(t) W_{0}^{T} P x(t) \\
& +x^{T}(t) P W_{1} l_{1}(t-h)+l_{1}^{T}(t-h) W_{1}^{T} P x(t) \\
& +e^{T}(t) P W_{2} \int_{t-\tau}^{t} l_{2}(s) d s+\left(\int_{t-\tau}^{t} l_{2}(s) d s\right)^{T} W_{2} P x(t) \\
\leq & \varepsilon_{1} l_{0}^{T}(t) l_{0}(t)+\varepsilon_{1}^{-1} x^{T}(t) P W_{0} W_{0}^{T} P x(t) \\
& +\varepsilon_{2} l_{1}^{T}(t-h) l_{1}(t-h)+\varepsilon_{2}^{-1} x^{T}(t) P W_{1} W_{1}^{T} P x(t) \\
& +\varepsilon_{3}\left(\int_{t-\tau}^{t} l_{2}(s) d s\right)^{T} \int_{t-\tau}^{t} l_{2}(s) d s+\varepsilon_{3}^{-1} x^{T}(t) P W_{2} W_{2}^{T} P x(t) \\
\leq & x^{T}(t)\left(\varepsilon_{1} G_{0}^{T} G_{0}+\varepsilon_{1}^{-1} P W_{0} W_{0}^{T} P+\varepsilon_{2}^{-1} P W_{1} W_{1}^{T} P+\varepsilon_{3}^{-1} P W_{2} W_{2}^{T} P\right) x(t) \\
& +\varepsilon_{2} x^{T}(t-h) G_{1}^{T} G_{1} x(t-h)+\varepsilon_{3}\left(\int_{t-\tau}^{t} l_{2}(s) d s\right)^{T} \int_{t-\tau}^{t} l_{2}(s) d s .
\end{aligned}
$$

Furthermore, from (7), (10) and Lemma 2, we have that

$$
\begin{aligned}
& \int_{t-\tau}^{t} e^{T}(s) Q_{2} x(s) d s \\
= & \varepsilon_{3} \tau \int_{t-\tau}^{t} e^{T}(s) G_{2}^{T} G_{2} x(s) d s \\
\geq & \varepsilon_{3} \tau \int_{t-\tau}^{t} l_{2}^{T}(s) l_{2}(s) d s \\
\geq & \varepsilon_{3}\left(\int_{t-\tau}^{t} l_{2}(s) d s\right)^{T} \int_{t-\tau}^{t} l_{2}(s) d s .
\end{aligned}
$$

Using (14) and the definition of $Q_{1}$ and $Q_{2}$ in (9) and (10), it indicates from (11) and (15) that

$$
\begin{aligned}
\frac{d \Phi(x(t))}{d t} \leq & x^{T}(t)\left\{-A^{T} P-P A+P\left(\varepsilon_{1}^{-1} W_{0} W_{0}^{T}+\varepsilon_{2}^{-1} W_{1} W_{1}^{T}+\varepsilon_{3}^{-1} W_{2} W_{2}^{T}\right) P\right. \\
& \left.+\varepsilon_{1} G_{0}^{T} G_{0}+\varepsilon_{2} G_{1}^{T} G_{1}+\varepsilon_{3} \tau^{2} G_{2}^{T} G_{2}\right\} x(t)
\end{aligned}
$$

which implies from the condition (6) that

$$
\frac{d \Phi(x(t))}{d t} \leq-\lambda_{\min }(-\Pi)|x(t)|^{2} .
$$


where

$$
\begin{aligned}
\Pi:= & -A^{T} P-P A+P\left(\varepsilon_{1}^{-1} W_{0} W_{0}^{T}+\varepsilon_{2}^{-1} W_{1} W_{1}^{T}+\varepsilon_{3}^{-1} W_{2} W_{2}^{T}\right) P \\
& +\varepsilon_{1} G_{0}^{T} G_{0}+\varepsilon_{2} G_{1}^{T} G_{1}+\varepsilon_{3} \tau^{2} G_{2}^{T} G_{2}<0 .
\end{aligned}
$$

It can now be concluded from Lyapunov stability theory that the dynamics of the neural network (3) is globally asymptotically stable. This completes the proof of Theorem 1 .

In Theorem 1, the stability analysis result (i.e., the stability criterion) is established in terms of a quadratic matrix inequality (6), which contains several scalar parameters. Obviously, it is generally difficult to solve such a quadratic matrix inequality since some tuning over the scalar parameters $\varepsilon_{1}>0, \varepsilon_{2}>0$ and $\varepsilon_{3}>0$ is needed. Fortunately, such a difficulty can be overcome by resorting to the linear matrix inequalities (LMIs). It should be mentioned that, in the past decade, LMIs have gained much attention for their computational tractability and usefulness in many areas, including the stability testing for neural networks [7], because the so-called interior point method (see [3]) has been proven to be numerically very efficient for solving the LMIs. Thus, our next goal is to restate Theorem 1 in terms of LMIs.

Theorem 2: If there exist three positive scalars $\varepsilon_{1}, \varepsilon_{2}, \varepsilon_{3}$ and a positive definite matrix $P \in \mathbb{R}^{n \times n}$ such that the following linear matrix inequality

$$
\left[\begin{array}{ccccccc}
-A P-P A & P W_{0} & \varepsilon_{1} G_{0}^{T} & P W_{1} & \varepsilon_{2} G_{1}^{T} & P W_{2} & \varepsilon_{3} \tau G_{2}^{T} \\
W_{0}^{T} P & -\varepsilon_{1} I & 0 & 0 & 0 & 0 & 0 \\
\varepsilon_{1} G_{0} & 0 & -\varepsilon_{1} I & 0 & 0 & 0 & 0 \\
W_{1}^{T} P & 0 & 0 & -\varepsilon_{2} I & 0 & 0 & 0 \\
\varepsilon_{2} G_{1} & 0 & 0 & 0 & -\varepsilon_{2} I & 0 & 0 \\
W_{2}^{T} P & 0 & 0 & 0 & 0 & -\varepsilon_{3} I & 0 \\
\varepsilon_{3} \tau G_{2} & 0 & 0 & 0 & 0 & 0 & -\varepsilon_{3} I
\end{array}\right]<0
$$

holds, then the dynamics of the neural network (3) is globally asymptotically stable.

Proof: Assume that the condition of this theorem is satisfied. Pre- and post-multiplying the inequality (18) by the block-diagonal matrix

$$
\operatorname{diag}\left\{I, \varepsilon_{1}^{-1 / 2} I, \varepsilon_{1}^{-1 / 2} I, \varepsilon_{2}^{-1 / 2} I, \varepsilon_{2}^{-1 / 2} I, \varepsilon_{3}^{-1 / 2} I, \varepsilon_{3}^{-1 / 2} I\right\}
$$

yield

$$
\left[\begin{array}{ccccccc}
\Omega_{1} & \varepsilon_{1}^{-1 / 2} P W_{0} & \varepsilon_{1}^{1 / 2} G_{0}^{T} & \varepsilon_{2}^{-1 / 2} P W_{1} & \varepsilon_{2}^{1 / 2} G_{1}^{T} & \varepsilon_{3}^{-1 / 2} P W_{2} & \varepsilon_{3}^{1 / 2} \tau G_{2}^{T} \\
\varepsilon_{1}^{-1 / 2} W_{0}^{T} P & -I & 0 & 0 & 0 & 0 & 0 \\
\varepsilon_{1}^{1 / 2} G_{0} & 0 & -I & 0 & 0 & 0 & 0 \\
\varepsilon_{2}^{-1 / 2} W_{1}^{T} P & 0 & 0 & -I & 0 & 0 & 0 \\
\varepsilon_{2}^{1 / 2} G_{1} & 0 & 0 & 0 & -I & 0 & 0 \\
\varepsilon_{3}^{1 / 2} W_{2} P & 0 & 0 & 0 & 0 & -I & 0 \\
\varepsilon_{3}^{-1 / 2} \tau G_{2} & 0 & 0 & 0 & 0 & 0 & -I
\end{array}\right]<0,
$$

or

$$
\left[\begin{array}{cc}
\Omega_{1} & \Omega_{3}^{T} \\
\Omega_{3} & -\Omega_{2}
\end{array}\right]<0
$$

where

$$
\begin{aligned}
& \Omega_{1}:=-A P-P A \\
& \Omega_{2}:=I \\
& \Omega_{3}:=\left[\begin{array}{llllll}
\varepsilon_{1}^{-1 / 2} P W_{0} & \varepsilon_{1}^{1 / 2} G_{0}^{T} & \varepsilon_{2}^{-1 / 2} P W_{1} & \varepsilon_{2}^{1 / 2} G_{1}^{T} & \varepsilon_{3}^{-1 / 2} P W_{2} & \varepsilon_{3}^{1 / 2} \tau G_{2}^{T}
\end{array}\right]^{T} .
\end{aligned}
$$


It follows from the Schur Complement Lemma (Lemma 1) that (20) holds if and only if

$$
\Omega_{1}+\Omega_{3}^{T} \Omega_{2}^{-1} \Omega_{3}<0
$$

or

$$
\begin{gathered}
-A P-P A+\varepsilon_{1}^{-1} P W_{0} W_{0}^{T} P+\varepsilon_{1} G_{0}^{T} G_{0}+\varepsilon_{2}^{-1} P W_{1} W_{1}^{T} P \\
+\varepsilon_{2} G_{1}^{T} G_{1}+\varepsilon_{3}^{-1} P W_{2} W_{2}^{T} P+\varepsilon_{3} \tau^{2} G_{2}^{T} G_{2}<0,
\end{gathered}
$$

which is (6). Hence, the proof follows from Theorem 1 immediately.

Remark 3: Notice that in Theorem 2, the matrix inequality (18) is linear on the parameters $\varepsilon_{1}>0, \varepsilon_{2}>0$, $\varepsilon_{3}>0$, and $P>0$. Therefore, by using the Matlab LMI toolbox, it is straightforward to check the feasibility of (18) without tuning any parameters, so as to determine global asymptotic stability of the neural network with both discrete and distributed delays. In contrast to the existing results based on matrix norm computation, such as those given in [1], [5], our LMI approach has the advantage that the LMIs can be solved numerically and effectively by using the interior-point method [3].

\section{NUMERICAL EXAMPLES}

Two simple examples are presented here in order to illustrate the usefulness of our main results. Our aim is to examine the global asymptotic stability of the give delayed neural networks.

Example 1. Consider a two-neuron neural network (3), where

$$
\begin{gathered}
A=\left[\begin{array}{cc}
0.9 & 0 \\
0 & 0.8
\end{array}\right], W_{0}=\left[\begin{array}{cc}
1 & -1.7 \\
-1.6 & 1
\end{array}\right], W_{1}=\left[\begin{array}{cc}
1 & 0.6 \\
0.5 & 0.8
\end{array}\right], W_{2}=\left[\begin{array}{ll}
0.4 & 0.3 \\
0.1 & 0.2
\end{array}\right], \\
G_{0}=\left[\begin{array}{cc}
0.1 & 0 \\
0 & 0.1
\end{array}\right], G_{1}=\left[\begin{array}{cc}
0.1 & 0 \\
0 & 0.1
\end{array}\right], G_{2}=\left[\begin{array}{cc}
0.2 & 0 \\
0 & 0.1
\end{array}\right], \tau=0.12 .
\end{gathered}
$$

By using the Matlab LMI toolbox, we solve the LMI (18) for $\varepsilon_{1}>0, \varepsilon_{2}>0, \varepsilon_{3}>0, P>0$ and obtain

$$
\varepsilon_{1}=1.1491, \quad \varepsilon_{2}=1.1483, \quad \varepsilon_{3}=1.1563, \quad P=\left[\begin{array}{lll}
0.2360 & 0.0805 \\
0.0805 & 0.2679
\end{array}\right] .
$$

Therefore, it follows from Theorem 2 that the two-neuron neural network (3) is globally asymptotically stable.

Example 2. Now, let us consider a third-order delayed neural network described by the following data

$$
\begin{gathered}
A=\left[\begin{array}{ccc}
2.3 & 0 & 0 \\
0 & 3.4 & 0 \\
0 & 0 & 2.5
\end{array}\right], W_{0}=\left[\begin{array}{ccc}
0.9 & -1.5 & 0.1 \\
-1.2 & 1 & 0.2 \\
0.2 & 0.3 & 0.8
\end{array}\right], \\
W_{1}=\left[\begin{array}{ccc}
0.8 & 0.6 & 0.2 \\
0.5 & 0.7 & 0.1 \\
0.2 & 0.1 & 0.5
\end{array}\right], W_{2}=\left[\begin{array}{ccc}
0.3 & 0.2 & 0.1 \\
0.1 & 0.2 & 0.1 \\
0.1 & 0.1 & 0.2
\end{array}\right], \\
\tau=0.12, G_{0}=G_{1}=G_{2}=0.2 I_{3}
\end{gathered}
$$

Again, by solving the LMI (18) for $\varepsilon_{1}>0, \varepsilon_{2}>0, \varepsilon_{3}>0$, and $P>0$, we obtain

$$
\begin{gathered}
\varepsilon_{1}=22.3438, \quad \varepsilon_{2}=22.0979, \quad \varepsilon_{3}=22.9265 \\
P=\left[\begin{array}{ccc}
4.2234 & 0.3039 & -0.0727 \\
0.3039 & 3.3450 & -0.1046 \\
-0.0727 & -0.1046 & 4.8751
\end{array}\right]
\end{gathered}
$$


which indicates that the delayed neural network (3) is globally asymptotically stable.

For Example 1 and Example 2, the responses of neuron dynamics to initial conditions are shown in Fig. 1 and Fig. 2, respectively. The simulation results imply that, in both examples, the neural network (3) is indeed globally asymptotically stable.

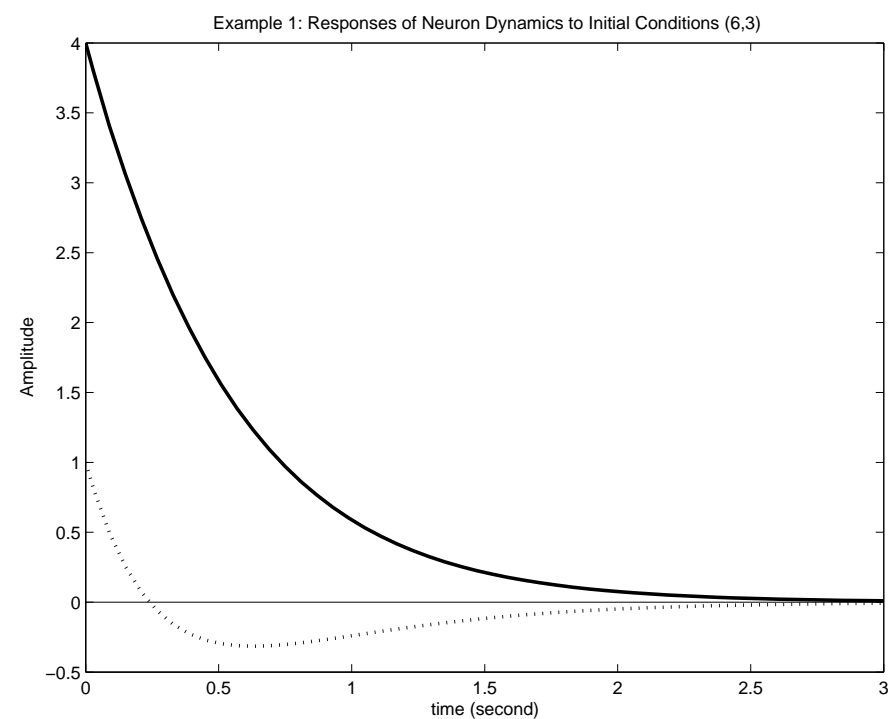

Fig. 1. $x_{1}$ (solid), $x_{2}$ (dotted).

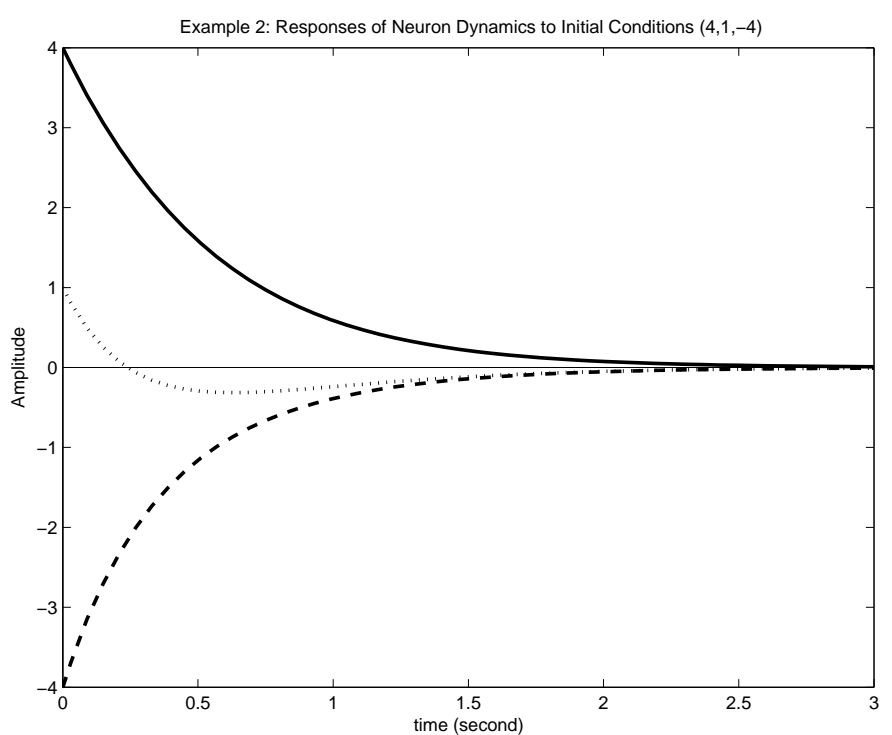

Fig. 2. $x_{1}$ (solid), $x_{2}$ (dotted), $x_{2}$ (dashed).

\section{Conclusions}

In this paper, we have dealt with the problem of global asymptotic stability analysis for a class of delayed neural networks, which involve both discrete and distributed time delays. We have removed the traditional monotonicity and smoothness assumptions on the activation function. A linear matrix inequality (LMI) approach has been developed to solve the problem addressed. The conditions for the global asymptotic stability have been derived in terms of the positive definite solution to an LMI involving several scalar parameters, and simple examples have been used to demonstrate the usefulness of the main results.

\section{REFERENCES}

[1] Arik, S. (2000). Stability analysis of delayed neural networks. IEEE Transactions on Circuits Systems -I, 47, $1089-1092$.

[2] Baldi, P., and Atiya, A. F. (1994). How delays affect neural dynamics and learning. IEEE Transactions on Neural Networks, 5, 612-621.

[3] Boyd, S., Ghaoui, L. EI, Feron, E., and Balakrishnan, V. (1994). Linear matrix inequalities in system and control theory. Philadephia: SIAM.

[4] Burton, T. (1985) Stability and periodic solution of ordinary differential equation and functional differential equations. Orlando, FL: Academic.

[5] Cao, J. (2000). Periodic oscillation and exponential stability of delayed CNNs. Physics Letters A, 270, $157-163$.

[6] Cao, J., Huang, D.-S., and Qu, Y. (2005) Global robust stability of delayed recurrent neural networks, Chaos, Solitons and Fractals, 23, 221-229.

[7] Cao, J. and Ho, D. W. C. (2005) A general framework for global asymptotic stability analysis of delayed neural networks based on LMI approach, Chaos, Solitons and Fractals, 24, 1317-1329.

[8] De Vries, B. and Principle, J. C. (1992) The gamma model - A new neural model for temporal processing. Neural Networks, $5,565-576$.

[9] Gu, K. (2000) An integral inequality in the stability problem of time-delay systems. In: Proceedings of 39th IEEE Conference on Decision and Control, December 2000, Sydney, Australia, 2805-2810.

[10] Hale, J. K. (1977) Theory of functional differential equations. New York: Springer-Verlag. 
[11] Huang, H., Cao, J., and Qu, Y. (2004) Global robust stability of delayed neural networks with a class of general activation functions, Journal of Computer and System Sciences, to appear.

[12] Joy, M. P. (1999b). On the global convergence of a class of functional differential equations with applications in neural network theory. Journal of Mathematical Analysis and Applications, 232, 61-81.

[13] Joy, M. P. (2000a). Results concerning the absolute stability of delayed neural networks. Neural Networks, $13,613-616$.

[14] Liang, J. and Cao, J. (2004) Global asymptotic stability of bi-directional associative memory networks with distributed delays. Applied Mathematics and Computation, 152, 415-424.

[15] Morita, M. (1993). Associative memory with nonmonotone dynamics. Neural Networks, 6(6), 115-126.

[16] Principle, J. C., Kuo, J.-M. and Celebi, S. (1994) An analysis of the gamma memory in dynamic neural networks. IEEE Trans. Neural Networks, 5(2), 337-361.

[17] Ruan, S. and Filfil, R. S. (2004) Dynamics of a two-neuron system with discrete and distributed delays. Physica D, 191, 323-342.

[18] Tank, D. W. and Hopfield, J. J. (1987) Neural computation by concentrating information in time. Proc. Nat. Acad. Sci., 84, 1896-1991.

[19] Van den Driessche, P., and Zou, X. (1998). Global attractivity in delayed Hopfield neural networks model. SIAM Journal of Applied Mathematics, 58, 1878-1890.

[20] Wang, Z., Ho, D. W. C. and Liu, X. (2005) State estimation for delayed neural networks. IEEE Transactions on Neural Networks, 16(1), 279-284.

[21] Xu, S., Lam, J. and Ho, D. W. C. (2004) Global robust exponential stability analysis for interval recurrent neural networks. Physics Letters A, 325, 124-133.

[22] Zhang, Y. (1996). Global exponential stability and periodic solutions of delay Hopfield neural networks. International Journal of System Sciences, 27, 227-231.

[23] Zhao, H. (2004) Global asymptotic stability of Hopfield neural network involving distributed delays. Neural Networks, 17, 47-53.

[24] Zhao, H. (2004) Existence and global attractivity of almost periodic solution for cellular neural network with distributed delays. Applied Mathematics and Computation, 154, 683-695.

[25] Zhao, H. (2005) Global exponential stability and periodicity of cellular neural networks with variable delays, Physics Letters A, 336, 331-341.

[26] Zhao, H., and Wang, G. (2004) Delay-independent exponential stability of recurrent neural networks, Physics Letters A, 333, 399-407.

[27] Zhao, H. (2003) Global stability of neural networks with distributed delays, Physical Review E, 68, art. no. 051909, 1-7.

[28] Zhao, H. (2002) Global stability of bidirectional associative memory neural networks with distributed delays, Physics Letters A, 297, 182-190. 